\title{
LONG-TERM NONCONJUGATE ADAPTATION OF HUMAN SACCADES TO ANISOMETROPIC SPECTACLES
}

\author{
H. G. Lemis and H. CollewisN \\ Department of Physiology I, Faculty of Medicine, Erasmus University Rotterdam, P.O. Box 1738, \\ 3000 DR Rotterdam, The Netherlands
}

(Recewed 29 June 1990; in revised form 4 February 1991)

\begin{abstract}
It is generally believed that saccades follow Hering's law in the sense that they are equally large in the two eyes. We demonstrated that saccades are different in size in the two eyes in 8 habitual wearers of anisometropic spectacles, which have lenses of different refractive powers, and therefore supply each eye with a differently sized visual image. The eye provided with the larger visual image made larger saccades than its fellow eye. This nonconjugate adaptation was almost complete for both horizontal and vertical saccades. Post-saccadic drift was also asymmetrically adapted: it reduced any fixation-disparity present at saccadic offset. The nonconjugate adaptation was also expressed in smooth-pursuit eye movements. In addition, these nonconjugate adaptations were present during monocular viewing, which shows that they were hard-programmed.
\end{abstract}

Hering's law Saccades Adaptation Aniseikonia Anisometropic spectacles Post-saccadic drift Smooth-pursuit

\section{INTRODUCTION}

Saccades are the rapid eye movements that allow us to voluntarily direct our gaze from one visual target to another. They bring newly selected targets to the fovea. It is generally assumed that the two eyes make saccades of equal size. This assumption is frequently referred to as Hering's law of equal innervation. According to this law, the two eyes receive the same innervation (Hering, 1868), or, in more current terms, the same motor commands. The ensuing conjugacy of saccades would, normally, be desirable, to maintain binocular foveation. Disruption of this parallelism between commands, saccades and binocular fixation would, however, be very likely to occur in a lifetime if the balance between the motor commands to the two eyes were indeed rigidly fixed. The slightest functional loss in one eye muscle would lead to unequal saccades in the two eyes, with diplopia as the probable result. Also the wearing of anisometropic spectacles, i.e. spectacles with lenses of unequal power, would cause this problem. Such spectacles produce visual images that are different in size for the two eyes because the lenses are positioned anterior to the nodal points of the eye. As a consequence, the angles of eccentricity of a peripheral target are different for the two eyes.
Binocular foveation of such targets requires eye movements of similarly different sizes, as the lenses do not move with the eyes.

An important question is, thus, whether the oculomotor system has the capability to adapt the motor commands asymmetrically for the two eyes, in order to maintain the functional yoking of the eyes. Erkelens, Collewijn and Steinman (1989) recently reported in detail on a subject who made saccades that were essentially different in size for the two eyes. This subject had been adapted for about 40 years to anisometropic spectacles. The nonconjugate saccades observed in the subject of Erkelens et al. (1989) reflected this difference and were, therefore, considered to result from an adaptation to the inequality in image-size: the eye presented with the larger visual image made larger saccades than its fellow eye. For vertical saccades, this long-term adaptation was almost perfect, i.e. the difference in saccadic amplitude between the two eyes was very close to what was called for by the spectacles. Horizontal saccades, however, were less perfectly adapted. Note, that such size-adaptation of eye movements can only partially correct for the size-difference in the two retinal images. It can, at best, preserve binocular foveation of the same point of an object despite the size difference between the retinal images of the object 
(aniseikonia), which is not reduced by such a strategy. Note also, that contact-lenses of different power do not induce a need for sizedifferences of the movements of the two eyes, because contact-lenses cause very little aniseikonia and, moreover, move with the eyes (Bennett \& Francis, 1962).

Less detailed reports on nonconjugate adaptations have been issued before by the same authors (Collewijn, Erkelens \& Steinman, 1988a), and also by Levi, Zee, Hain, Fletcher and Miller (1988), Schor, Gleason and Horner (1988), Horner, Gleason and Schor (1988), and by Zee and Levi (1989). Schor, Gleason and Horner (1990) recently published an extensive experimental study on short-term adaptation, while Oohira, Zee and Guyton (1991) recently studied nonconjugate adaptation in one case of long-standing anisometropic correction. This recent, specific interest in nonconjugate adaptations of saccades to the wearing of anisometropic spectacles follows earlier studies on more general aspects of saccadic plasticity. It had been shown before that the saccadic subsystem is capable of adapting adequately to various disruptions of saccadic metrics, such as physical damage to one or more external eyemuscles. These adaptations restored, to a considerable extent, the accuracy of saccadic eye movements, which had suffered strongly from the sudden muscle-weakness. Adaptation occurred in response to either damage caused by disease in humans (Kommerell, Olivier \& Theopold, 1976; Abel, Schmidt, Dell'Osso \& Daroff, 1978; Optican, Zee \& Chu, 1985), or experimental damage in monkeys (Optican \& Robinson, 1980; Snow, Hore \& Vilis, 1985; Virre, Cadera \& Vilis, 1988). Appropriate saccadic adaptations were also demonstrated in response to targets that jump consistently, during the execution of a saccade, in a specific direction, thereby requiring adaptive changes in saccadic magnitude or in saccadic direction (Miller, Anstis \& Templeton, 1981; Deubel, Wolf \& Hauske, 1986; Deubel, 1987; Albano \& King, 1989). Except for the experiments on monkeys by Snow et al. (1985) and Virre et al. (1988), who weakened external eye-muscles of only one eye, all the reported adaptations were, in essence, conjugate, i.e. equally large for the two eyes.

The experiments described above offer impressive examples of how well the saccadic subsystem may adapt to a variety of stimuli. However, saccadic adaptation is presumably not restricted to such experimental conditions, but may take place continuously in response to the wear and tear of every-day life. Any change in the saccadic system, caused by the effects of, for example, growth, ageing, disease, injury or fatigue may jeopardize saccadic control. Experiments on saccadic plasticity may help to increase our understanding of how the saccadic system copes with such changes, and maintains a high quality of saccadic control throughout a lifetime. Experiments in which external eye-muscles are severed may resemble the sudden effects of acute muscle-pareses. They mimic only poorly, however, the more insidious changes that will normally take place. As these changes may have variable time-courses and are likely to occur haphazardly within the oculomotor system at various locations, nonconjugate adaptations are more likely to be required than conjugate adaptations. Fairly moderate and asymmetrical challenges, such as anisometropic spectacles, are therefore probably useful in studying the adaptive properties of the saccadic system. Since the experiments on long-term nonconjugate adaptation by Erkelens et al. (1989) were conducted on a single subject, we recorded and analysed saccades of a much larger number of habitual wearers of anisometropic spectacles. In the present experiments, we have systematically addressed several questions.

As already mentioned, Erkelens et al. (1989) found that nonconjugate adaptations were more complete for vertical saccades than for horizontal saccades. These authors suggested that the difference in the degree of adaptive change for the two meridians might be associated with differences in the fusional limits for either meridian. Fusional limits for the vertical meridian are smaller than those for the horizontal meridian (Fender \& Julesz, 1967; Piantanida, 1986; Erkelens, 1988). Nonconjugate adaptation would, possibly, only occur up to the level that fusion were just possible, and thus be more perfect in the vertical meridian. However, in the present experiments we shall demonstrate that long-term nonconjugate adaptations may be better along either the horizontal or the vertical meridian.

Deubel et al. (1986) observed that the adaptive changes of saccades that were induced by intrasaccadic target displacements at a specific saccade-size, were also reflected in the magnitude of saccades of a different size in the same direction. From this observation Deubel et al. (1986) concluded that adaptation takes place in a simple, parametric manner: one single gain element would determine the magnitude of 
adaptive changes in saccadic sizes for all target eccentricities. A similar notion of a simple, direction-specific, gain element had been suggested previously by Miller et al. (1981). However, the experiments of Erkelens et al. (1989), as well as our present observations, do not support such parametric adjustment.

Finally, Erkelens et al. (1989) briefly mentioned a very small adaptive change in postsaccadic drift, secondary to the long-term wearing of anisometropic spectacles. This contrasts with the marked post-saccadic drift associated with saccadic adaptations to palsies of one or more external eye muscles, either caused by disease or brought about experimentally (Kommerell et al., 1976; Abel et al., 1978; Optican \& Robinson, 1980; Optican et al., 1985; Snow et al., 1985). We shall, therefore, also focus on plastic changes in post-saccadic drift, secondary to the long-term wearing of anisometropic spectacles. Furthermore, nonconjugate adaptations of smooth-pursuit eye movements will be dealt with in passing. The present investigations formed part of a doctoral thesis (Lemij, 1990).

\section{METHODS}

\section{Subjects}

Nine habitual wearers of anisometropic spectacles participated in these experiments. None of them had any history of ocular or oculomotor pathology. All of them had different refractive anomalies in the two eyes, which had been corrected by anisometropic spectacles for many years. Visual acuities were $5 / 5$ or better for either eye in all subjects wearing their habitual corrections. The anisometropias were, on average, $2 \mathrm{D}$ or more. In one subject (PA) the anisometropia was less than $1 \mathrm{D}$ in the horizontal meridian, but more than $2 \mathrm{D}$ in the vertical meridian. Two subjects ( $\mathrm{CR}$ and $\mathrm{CB}$ ) normally wore their spectacles all day through, whereas all other subjects used to wear them only several hours a day. The individual lens-prescriptions at the time of the experiments are presented, for all subjects, in Table 1, which also shows the age and sex of each subject, as well as the time of wearing of the spectacles, both in terms of the number of years and the amount of time per day during the last 3 months prior to the experiments. In addition, 9 subjects (age 25-44 years) who wore no spectacles and had no history of ocular or oculomotor pathology participated in these experiments as controls. Among them were 4 myopes, who wore corrective, rigid contact lenses normally and also during the experiment. Visual acuity was at least $5 / 5$ in all emmetropic and corrected myopic control subjects.

Because the presence of binocular vision was considered important for the outcome of the experiment, stereopsis was assessed in all subjects, both spectacle-wearers and controls, with a standard test (type: TNO test for stereoscopic vision). All subjects passed this test (criterion: $60 \mathrm{sec}$ arc or better)

\section{Stimuli}

Our main objective was to study differential adaptation of saccadic movements of the two eyes to anisometropic spectacles. To avoid confusion with vergence eye movements, we had to employ stimuli that did not require any vergence changes. As discussed by Collewijn, Erkelens and Steinman (1988b), any circle running through the centres of rotation of the two eyes forms an iso-vergence locus: any point on that circle which is binocularly foveated requires exactly the same angle of horizontal vergence for the two eyes. If the iso-vergence circle is rotated around an axis that passes through the centres of rotation of the two eyes, it describes a toroid surface, which contains also vertical iso-vergence loci, if eye-positions are expressed in a Helmholtz

Table 1. Lens-prescriptions and additional data of subjects

\begin{tabular}{|c|c|c|c|c|c|c|}
\hline \multirow[b]{2}{*}{$\mathbf{S}$} & \multirow[b]{2}{*}{ Age } & \multirow[b]{2}{*}{ Sex } & \multicolumn{2}{|c|}{ Wearing time } & \multirow[b]{2}{*}{ Prescription left lens } & \multirow[b]{2}{*}{ Prescription right lens } \\
\hline & & & Years & Day & & \\
\hline $\mathbf{K H}$ & 55 & $\mathrm{~F}$ & \pm 40 & $\mathrm{SH}^{*}$ & $\mathrm{~S}-1.25$ & $\mathrm{~S}-3.75, \mathrm{C}-1.0$, axis $90 \mathrm{deg}$ \\
\hline CR & 73 & $\mathrm{~F}$ & 51 & $\mathrm{AD}^{*}$ & $\mathrm{~S}-2.75, \mathrm{C}-0.75$, axis $170 \mathrm{deg}$ & $\mathrm{S}+0.75, \mathrm{C}-1.0$, axis $10 \mathrm{deg}$ \\
\hline PJ & 46 & $\mathbf{M}$ & \pm 35 & SH & $S+1.0$ & $\mathrm{~S}-2.25, \mathrm{C}-0.5$, axis $155 \mathrm{deg}$ \\
\hline PA & 39 & $\mathrm{~F}$ & 25 & SH & $\mathrm{S}-1.25, \mathrm{C}-2.0$, axis $85 \mathrm{deg}$ & $\mathrm{S}+1.5, \mathrm{C}-5.0$, axis $80 \mathrm{deg}$ \\
\hline CB & 46 & $\mathrm{~F}$ & 42 & $A D$ & $S-2.0$ & $\mathrm{~S}-9.0, \mathrm{C}+5.0$, axis $65 \mathrm{deg}$ \\
\hline MB & 41 & $\mathrm{~F}$ & 32 & SH & $\mathrm{S}-1.25, \mathrm{C}-1.5$, axis $10 \mathrm{deg}$ & $\mathrm{S}-5.0, \mathrm{C}-1.25$, axis $10 \mathrm{deg}$ \\
\hline GP & 48 & $\mathrm{~F}$ & 2 & SH & $\mathrm{S}+3.5, \mathrm{C}-1.5$, axis $10 \mathrm{deg}$ & $S+0.25$ \\
\hline MJ & 33 & $\mathrm{~F}$ & 19 & $\mathrm{SH}$ & $\mathrm{S}-3.0, \mathrm{C}-0.5$, axis $100 \mathrm{deg}$ & $\mathrm{S}-1.0, \mathrm{C}-0.5$, axis $70 \mathrm{deg}$ \\
\hline $\mathrm{HH}$ & 61 & $\mathbf{M}$ & \pm 40 & SH & $S-3.5, C-1.0$, axis $100 \mathrm{deg}$ & $S+0.25$ \\
\hline
\end{tabular}

*SH, several hours a day; AD, all day through. 
coordinate system, because the plane of regard in Helmholtz's coordinate system coincides with the plane of an iso-vergence circle (for a summary of Helmholtz's coordinate system, see Carpenter, 1988). Such a toroid surface was constructed from glassfibre and polyester, and coated with white paint. This construction served as the screen for stimulus presentation. The diameter of the iso-vergence circle was $80 \mathrm{~cm}$. In a properly placed subject, the screen covered almost the entire visual field. Subjects were positioned with reference to their corneal vertices, which were assumed to lie $13.5 \mathrm{~mm}$ anterior to the centres of rotation of their eyes (Alpern, 1962). Head movements of the subjects were restricted by adjustable forehead- and chinsupports and straps around the head.

The targets consisted of two bright-red He-Ne laser spots with a diameter of $5 \mathrm{~min}$ arc. For each target, a laser beam was first projected onto a front-surface mirror, which was mounted on the axle of a galvanometer (General Scanning). This axle was positioned horizontally, so that, by rotation of the axle the beam could be shifted in a vertical plane. Next, the beam was reflected onto the screen by a second front-surface mirror, which was mounted on a vertically positioned axle which allowed for beam-shifts in a horizontal plane. This axle was positioned on the iso-vergence circle that was described above, to allow for a simple control of the horizontal target position. Two of such sets (of two mirrors each) were mounted with one set on each side of the subject's head. All four scanners were driven independently by a computer. We therefore had two targets that were independently controlled and could be positioned anywhere on the screen. Position was controlled by an accuracy of better than $1 \%$. Each scanner had a built-in transducer which supplied analog output signal representing the veridical angular positions of the mirrors. If necessary, one target could be extinguished.

\section{Eye movement recording and calibration}

Movements of both eyes were recorded simultaneously by magnetic sensor-coils, introduced by Robinson (1963) and modified by Collewijn, Van der Mark and Jansen (1975). Two magnetic fields, in spatial and phase quadrature, were employed in a Robinson-configuration (Robinson, 1963). The field frequency was $1245 \mathrm{~Hz}$. Decomposition into two analog voltages, representing vertical and horizontal eye-positions, was done by dual-phase lock-in amplifiers (EG \& G Princeton Applied Research, model 5210).
Target-positions were recorded simultaneously with the eye-positions. All position signals were subsequently low-pass filtered at a cut-off frequency of $125 \mathrm{~Hz}$, digitized with 12-bit precision and sampled at a rate of $238 \mathrm{~Hz}$. All recordings were stored on disk or tape for off-line analysis. The overall noise level was less than $1.5 \mathrm{~min}$ arc.

The recording-equipment was pre-calibrated for an average sensor-coil. To prevent any artificial nonconjugacies in the recordings of concomitant saccades of the two eyes caused by unequal sensitivities of the coils, e.g. due to an extra turn in one coil, every individual coil was carefully recalibrated in its final position, i.e. on the eye of the properly positioned subject. To speed up the experimental procedures, these secondary fine-tunings were carried out off-line, based on calibration trials, recorded during monocular fixations of targets with known positions. To that end we had our subjects, after completing the actual experiments, take off their spectacles and then monocularly fix, with either eye, 8 bright-red targets ( 4 along the horizontal and 4 along the vertical meridian) at standard positions. The subjects were requested to carry out these monocular fixations, lasting $4 \mathrm{sec}$ each, as accurately as possible. The mean sensitivity and the mean offset, computed from the recordings of these monocular fixations, were considered to represent their veridical values. These values were subsequently fed into a special correction program which digitally recalibrated all recordings. These recalibrations were maximally on the order of $5 \mathrm{deg}$ for offset-corrections, necessitated by off-centre positioning of the coils on the eyes, and $2 \%$ for sensitivity-corrections. The same computer program transformed both the eye-positions and the target-positions into Helmholtz's coordinates. These coordinates could, for practical reasons, not be obtained directly. The same procedure was followed for our control experiments.

\section{Experimental procedures and data analysis}

During the experiments, all subjects wore their own anisometropic spectacles. The two brightred $\mathrm{He}-\mathrm{Ne}$ laser spots were simultaneously projected onto the screen. These two spots served as stationary targets. We used stationary targets because they yield more accurate saccades than a jumping target (Lemij \& Collewijn, 1989). The room lights were left on to provide a dim background illumination of the screen. Subjects were requested to make accurate gaze shifts between the two stationary targets at a comfort- 
able pace of $45 / \mathrm{min}$, as marked by a ticking sound from a loudspeaker. We did not exert any time-pressure, to avoid a possible compromise between accuracy and speed. Subjects were also asked to refrain from blinking during actual recording, because blinking produces disjunctive eye movements (Collewijn, van der Steen \& Steinman, 1985). Each experiment consisted of 24 trials for each subject. One half of these trials involved horizontal saccades, the other half vertical saccades. In each meridian, saccades were made between targets that were $5,10,20$ or $30 \mathrm{deg}$ apart, positioned symmetrically around the straight-ahead position. Viewing was either binocular, or monocular with either eye, in equal numbers of trials. The order of the trials was randomized for every subject. To obtain monocular viewing, subjects covered one eye with a hand-held patch that did not limit the visual field of the viewing, fellow eye. Each trial lasted $12 \mathrm{sec}$ and contained 8-10 saccades. Before any trial began, the targets were presented in their new positions and subjects were allowed to practise for a few seconds. When the subjects felt ready for the trial, they started the actual recording by pushing a start button. This procedure was chosen, because we were interested in steady-state performance, not in transient effects related to sudden changes in the visual stimuli.

After all 24 saccade-trials had been completed, one target was extinguished, and the other target made a smooth, circular movement, with a diameter of $30 \mathrm{deg}$ and a velocity of $11 \mathrm{deg} / \mathrm{sec}$. Subjects were instructed to follow the target as accurately as possible for $12 \mathrm{sec}$, beginning at the push of the start button. Viewing was either binocular, or monocular with either eye. These recordings were made to study nonconjugate adaptations of smooth-pursuit eye movements to the wearing of anisometropic spectacles.

The recordings were analyzed by computer programs. Saccades were identified by the following standard criteria: (1) the velocity of the eye movements exceeded $15 \mathrm{deg} / \mathrm{sec}$, and (2) the movement was also larger than a threshold amplitude, which was set at 2 deg for the smallest target separation ( $5 \mathrm{deg}$ ) and $4 \mathrm{deg}$ for all other target separations $(10-30 \mathrm{deg})$. When eye velocity fell below $15 \mathrm{deg} / \mathrm{sec}$, the saccade was considered to have ended (saccadic offset). Each saccade was characterized by its magnitude and direction. In addition, the mean velocity of the post-saccadic drift was calculated as the mean velocity of the eye movement over a period of
$68 \mathrm{msec}$, beginning $25 \mathrm{msec}$ after saccadic offset. These criteria were adopted first of all to disregard eye movements associated with dynamic overshoot (Bahill, Clark \& Stark, 1975; Kapoula, Robinson \& Hain, 1986), and secondly to avoid contamination of the computations by secondary saccades. As was demonstrated before (Lemij \& Collewijn, 1989), virtually all secondary saccades occur well over $90 \mathrm{msec}$ after saccadic offset.

The degree of nonconjugacy was expressed by the difference in saccadic size between concomitant saccades of the two eyes. For the spectacle-wearers this size-difference was defined as the magnitude of the saccade of the eye that was required to make the larger saccade, as imposed by the spectacle anisometropia, minus the magnitude of the concurring saccade of the fellow eye. For the control subjects saccadic size-differences were, arbitrarily, calculated as the magnitude of a saccade of the right eye minus the magnitude of the concomitant saccade of the left eye. We used size-differences of concurring saccades, instead of saccadic sizes per se, as a measure of the degree of nonconjugacy for two reasons: (1) the occurrence of saccadic undershoot would make it difficult to determine the degree of nonconjugacy by comparing the actual saccadic size with the required gaze-shift of each eye, and (2) the variability of saccadic size of each eye, expressed in standard deviations, would be a meaningless measure of nonconjugate adaptation, as opposed to the variability of differences in saccadic size.

The number of data was balanced for the two meridians, the four target separations, the three viewing conditions and the two directions of the saccades for either meridian. For statistical analysis, these data were submitted to a statistical computer program (SPSS-X), which was used to compute mean values and also to test any observed differences by means of a multivariate analysis of variance (MANOVA). To meet the requirements of normal distributions and homogeneous variances, the differences in saccadic size between the two eyes, as well as the postsaccadic drift-velocities were transformed logarithmically. As it turned out that one subject (GP) had cooperated poorly, by frequently not looking at the appropriate visual targets, her data were disregarded.

As a final comment on our methods, some displacement of the spectacles on the head was likely to occur during the running of the present experiments. This would consequently affect the position of the targets as viewed through the 
spectacles. Therefore, it was not feasible to assess where the targets were seen at all times by each of the two eyes. For this reason, we refrained from measuring position-errors at saccadic offset. We shall, for the same reason, also refrain from plotting target-positions in any of the figures.

\section{RESULTS}

\section{Saccadic size}

In all spectacle-wearers, the saccades of the two eyes were significantly different in size $\left(F_{1,7}=83.3 ; P<0.0005\right)$. These nonconjugacies were present in horizontal, as well as in vertical saccades, both during binocular and monocular viewing. The size differences always had the direction required to match the size differences induced by the spectacles. Our results confirm similar findings by Erkelens et al. (1989). The nonconjugacies could be as large as about $3 \mathrm{deg}$. These results are at variance with Hering's law of equal innervation in the sense that the two eyes made saccadic movements that were different in size. In contrast, saccades of the 9 control subjects were well yoked. Their saccadic sizedifferences were, on average, about $0.1 \mathrm{deg}$ (SD: $0.5 \mathrm{deg}$ ) for horizontal saccades and about 0.1 $\operatorname{deg}$ (SD: $0.3 \mathrm{deg}$ ) for vertical saccades. Similarly perfect yoking has been found in normal subjects by Collewijn et al. (1988b, c).

As will be shown in more detail further on, the size differences of the saccades of our spectaclewearers adequately met the requirements of their spectacles. Therefore, we attribute these size-differences to adaptation.

Figure 1 presents typical recordings of concomitant, nonconjugately adapted, saccades of both eyes in either meridian during monocular viewing (right eye covered), for a target separation of $30 \mathrm{deg}$ (subject $\mathrm{CR}$; right panels). For comparison with normal, unadapted saccades, typical binocular recordings of a control subject have been added to the figure (left panels). The control subject also viewed with the left eye only. In this case, the size differences between saccades of subject CR's two eyes were on the order of $1.7 \mathrm{deg}$ for horizontal saccades and $2.6 \mathrm{deg}$ for vertical saccades, at a nominal target separation of $30 \mathrm{deg}$. It is evident from Fig. 1 that the saccades of the control subject were, in contrast, virtually equal in size. We emphasize that these recordings were made during monocular viewing, i.e. in the absence of a direct pressure for any saccadic nonconjugacy in either subject. This demonstrates that the nonconjugate adaptations in subject $\mathrm{CR}$ were hard-programmed.
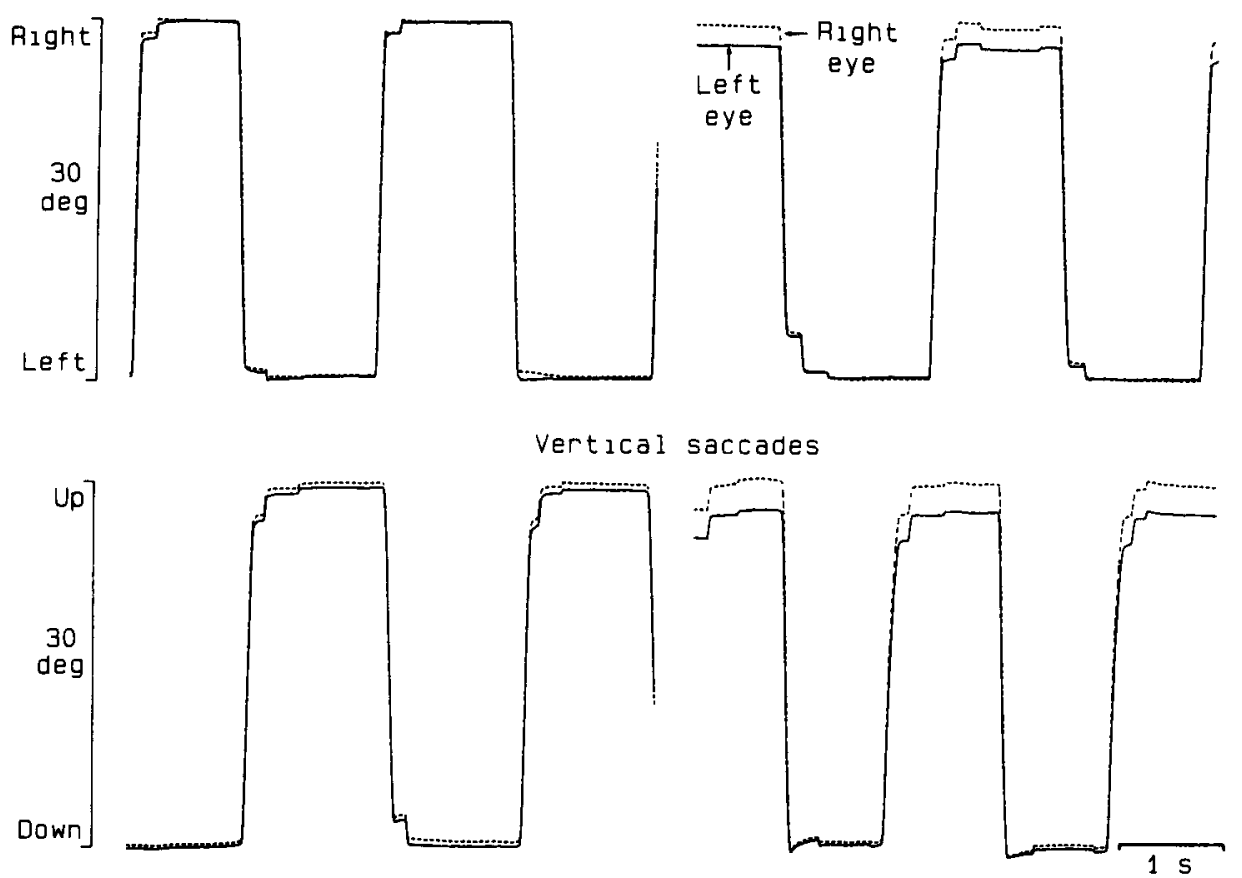

Fig. 1. Typical recordings of binocular saccades in a control subject who wore no spectacles (left panels) and nonconjugate saccades in subject $\mathrm{CR}$, who wears anisometropic spectacles (right panels). Recordings were made with only the left eye viewing. Nominal target separation: $30 \mathrm{deg}$. Continuous lines: left eye position; dotted lines: right eye position. 
Table 2. Mean differences in saccadic size (+SD) between the two eyes (deg)

\begin{tabular}{lcccccc}
\hline & & \multicolumn{2}{c}{ Horizontal saccades } & & \multicolumn{2}{c}{ Vertical saccades } \\
\cline { 7 - 8 } \cline { 6 - 7 } S. & $\begin{array}{c}\text { Target } \\
\text { sep. (deg) }\end{array}$ & Monoc. view & Binoc. view & & Monoc. view & Binoc. view. \\
\hline Contr. & 10 & $0.15(0.42)$ & $0.10(0.33)$ & & $0.06(0.19)$ & $0.04(0.26)$ \\
& 30 & $0.08(0.65)$ & $0.06(0.42)$ & & $0.29(0.33)$ & $0.04(0.25)$ \\
KH & 10 & $0.56(0.16)$ & $0.93(0.24)$ & & $0.47(0.26)$ & $0.68(0.31)$ \\
& 30 & $0.68(0.55)$ & $1.91(0.20)$ & & $1.21(0.42)$ & $1.36(0.31)$ \\
CR & 10 & $0.26(0.48)$ & $0.49(0.24)$ & & $0.63(0.20)$ & $0.56(0.31)$ \\
& 30 & $1.33(0.37)$ & $1.75(0.47)$ & & $2.14(0.41)$ & $2.11(0.14)$ \\
PJ & 10 & $0.56(0.71)$ & $0.79(0.34)$ & & $0.22(0.14)$ & $0.51(0.14)$ \\
& 30 & $1.66(1.13)$ & $2.86(0.37)$ & & $0.97(0.23)$ & $2.45(0.49)$ \\
PA & 10 & $-0.04(0.20)$ & $0.05(0.05)$ & & $0.38(0.13)$ & $0.43(0.07)$ \\
& 30 & $0.34(0.38)$ & $0.53(0.21)$ & & $1.57(0.44)$ & $1.61(0.25)$ \\
CB & 10 & $0.08(0.28)$ & $0.31(0.20)$ & & $0.78(0.14)$ & $0.80(0.22)$ \\
& 30 & $0.71(0.63)$ & $1.53(0.36)$ & & $2.48(0.95)$ & $2.93(0.21)$ \\
MB & 10 & $0.55(0.45)$ & $0.71(0.22)$ & & $0.40(0.19)$ & $0.34(0.22)$ \\
& 30 & $1.41(0.52)$ & $1.98(0.10)$ & & $0.71(0.35)$ & $1.23(0.34)$ \\
MJ & 10 & $0.19(0.18)$ & $0.15(0.18)$ & & $0.13(0.13)$ & $0.36(1.12)$ \\
& 30 & $0.91(0.48)$ & $0.64(0.37)$ & & $0.65(0.33)$ & $0.61(1.18)$ \\
HH & 10 & $0.31(0.62)$ & $0.61(0.66)$ & & $0.18(1.44)$ & $0.51(0.27)$ \\
& 30 & $1.61(0.86)$ & $3.16(0.40)$ & & $1.20(0.30)$ & $1.64(0.53)$ \\
\hline
\end{tabular}

The mean size-differences between saccades of the two eyes for every subject are shown in Table 2. Distinctions were made between horizontal and vertical saccades, and between monocular and binocular viewing. For simplicity, only the data relating to the 10 and $30 \mathrm{deg}$ target separations have been tabulated. The pooled data of our control subjects have also been added to Table 2. In the spectacle-wearers, saccadic size-differences between the two eyes generally increased with the saccadic amplitude $\left(F_{3,21}=\right.$ $61.3 ; P<0.0005$ ), in agreement with the requirements of the spectacles. In addition, the saccadic size-differences of the spectacle-wearers were generally larger during binocular viewing than during monocular viewing $\left(F_{2,14}=7.48 ; P<\right.$ $0.01)$. This was true for both horizontal and vertical saccades. We did not observe any differences in nonconjugate adaptation between those subjects who wore their spectacles all day through and those who wore them intermittently.

Although hard-programmed nonconjugate adaptations are better reflected by saccadic size-differences between the two eyes during monocular viewing than during binocular viewing, we shall nonetheless also discuss the nonconjugacies that occurred during binocular viewing, because those reflect how well the eye movements were adapted to normal, i.e. binocular, viewing conditions. The size-difference between horizontal saccades of the two eyes could become as large as about $3.2 \mathrm{deg}$ (subject $\mathrm{HH}$ ) during binocular viewing (nominal target separation: $30 \mathrm{deg}$ ). During monocular viewing, however, this size-difference was considerably smaller and amounted to maximally about 1.6 deg (subjects $\mathrm{HH}$ and PJ). For vertical saccades, maximal saccadic size-differences between the two eyes were about $2.9 \mathrm{deg}$ (subject CB) during binocular viewing and 2.5 deg (same subject) during monocular viewing (nominal target separation: $30 \mathrm{deg}$ ). In subject PA the sizedifferences between horizontal saccades of the two eyes were very small, in agreement with the small horizontal anisometropia of her spectacles (cf. Table 2 with Table 1). Her differences in size of saccades made along the horizontal meridian were actually similar to those of our control subjects. Along the vertical meridian, however, her saccades were considerably different in size (Table 2).

Having demonstrated nonconjugacies in saccadic size that had the proper direction to be adaptive to the anisometropic spectacles, we next examined how complete these nonconjugate adaptations were. We first determined the saccadic size-differences that were actually required by the spectacles. To that end we calculated the angular target separations as viewed by every individual eye of our spectacle-wearers from recordings made during steady, monocular fixations of the targets with either eye, while the spectacles were worn. The difference in gaze-shift amplitude between the two eyes was considered as the required difference in saccadic size between the two eyes. These required sizedifferences amounted, on average, to about $2.4 \%$ for every dioptre of anisometropia, when 
the orientations of the cylinder-axes had been taken into account. However, this percentage was very variable and ranged between a minimum of about $1.2 \%$ and a maximum of about $3.4 \%$. We attribute this variability largely to the various distances between the spectacle-lenses and the centres of rotation of the eyes among the different subjects. Thus, the nominal anisometropia of the spectacles in dioptres proved to be only a rough indicator of the required sizedifferences between saccades of the two eyes. For the control subjects the required saccadic size-difference was, by definition, 0 deg at every target separation.

Thereafter, we subtracted the actual sizedifference between concomitant saccades of the two eyes from the required size-difference. This difference between required and actual saccadic size-difference between the two eyes, corresponding to a fixation-disparity at saccadic offset, will be alluded to briefly as the vergence-deficit in this paper. This term (which is used in a purely technical sense, without implying any role of a vergence-subsystem in the adaptation) will be used as a measure of the amount that nonconjugate adaptation fell short of what was actually required by the spectacles.

In general, nonconjugate adaptation was more complete during binocular viewing than during monocular viewing. In our spectacle-wearers, the vergence-deficit of horizontal saccades was almost always less than $1.0 \mathrm{deg}$ during binocular viewing (mean value: $0.26 \mathrm{deg}$; SD: $0.40 \mathrm{deg}$ ). In one subject it could become as large as $1.2 \mathrm{deg}$ (subject MB; nominal target separation: $30 \mathrm{deg}$ ). During monocular viewing the vergence-deficit at the end of horizontal saccades was, on average, more than twice as large $(0.60 \mathrm{deg} ;$ SD: $0.75 \mathrm{deg})$ as during binocular viewing, with maximum values of $1.9 \mathrm{deg}$ (subject $\mathrm{HH}$; nominal target separation: $30 \mathrm{deg}$ ). Vertical saccades also showed smaller vergence-deficits during binocular viewing than during monocular viewing $\left(F_{2,14}=7.5 ; P<0.01\right)$; mean values were $0.43 \mathrm{deg}$ (SD: $0.54 \mathrm{deg}$ ) for binocular viewing and $0.60 \mathrm{deg}$ (SD: $0.80 \mathrm{deg}$ ) for monocular viewing. Maximum values of vergence-deficits associated with vertical saccades were $1.7 \mathrm{deg}$ (subject $\mathrm{HH}$ ) and $2.9 \mathrm{deg}$ (subject PJ) for binocular and monocular viewing, respectively. These large vergencedeficits were associated with our largest target separation (nominally $30 \mathrm{deg}$ ). Vergence-deficits gradually increased with the target separation $\left(F_{3,21}=12.6 ; P<0.0005\right)$. This increase was more prominent during monocular viewing than during binocular viewing $\left(F_{6,42}=4.81 ; P<\right.$ 0.0005 ). The vergence-deficits of vertical saccades were, for all subjects taken together, not consistently different from the vergence-deficits of horizontal saccades $\left(F_{1,7}=0.18 ; P>0.5\right)$, either during binocular, or during monocular viewing. However, within some subjects, the vergencedeficits associated with saccades in one meridian (either horizontal or vertical) were consistently larger than the vergence-deficits of saccades in the orthogonal meridian. These differences between horizontal and vertical vergence-deficits were not clearly correlated with differences between the anisometropias along these two meridians.

To summarize these data, individual means of saccadic size-differences have been plotted as a function of the required differences in saccadic size in Fig. 2 (binocular viewing) and Fig. 3 (monocular viewing). Perfect adaptations would fall along the $45 \mathrm{deg}$ diagonals (interrupted lines). Because there were no significant differences between horizonal and vertical saccades with respect to the actual saccadic size-differences between the two eyes, saccades along both meridians have been pooled. During binocular viewing (Fig. 2), the overall average degree of adaptation was about $74 \%$, as indicated by the slope of a linear regression (continuous line). During monocular viewing, however, the overall average of nonconjugate adaptation dropped to about $46 \%$ of what was required. This

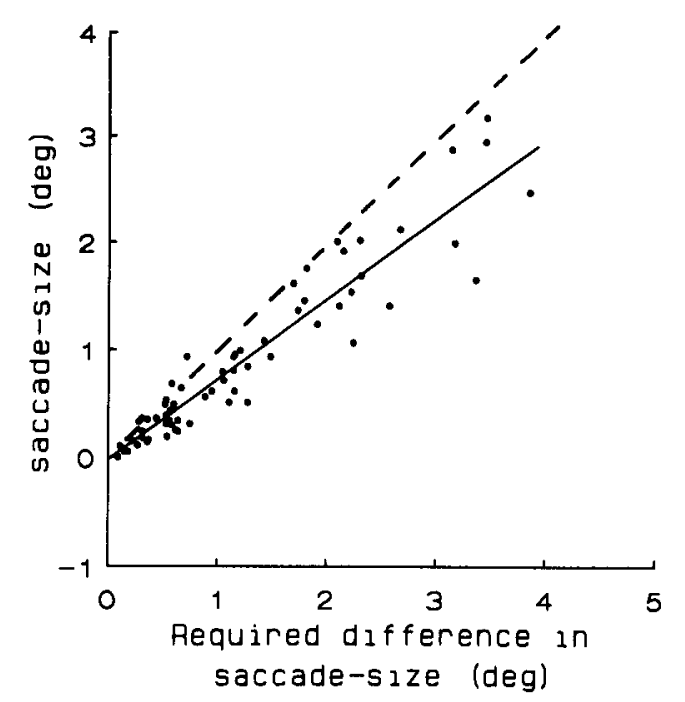

Fig. 2. Actual (signed) difference in saccadic size between the two eyes, plotted as a function of the required difference in saccadic size for all four subjects and both meridians. Data relate to binocular viewing; horizontal and vertical saccades have been pooled. The interrupted line (slope $=1.0$ ) represents complete adaptation. The continuous line (slope 0.74) represents the linear regression on the pooled data. 


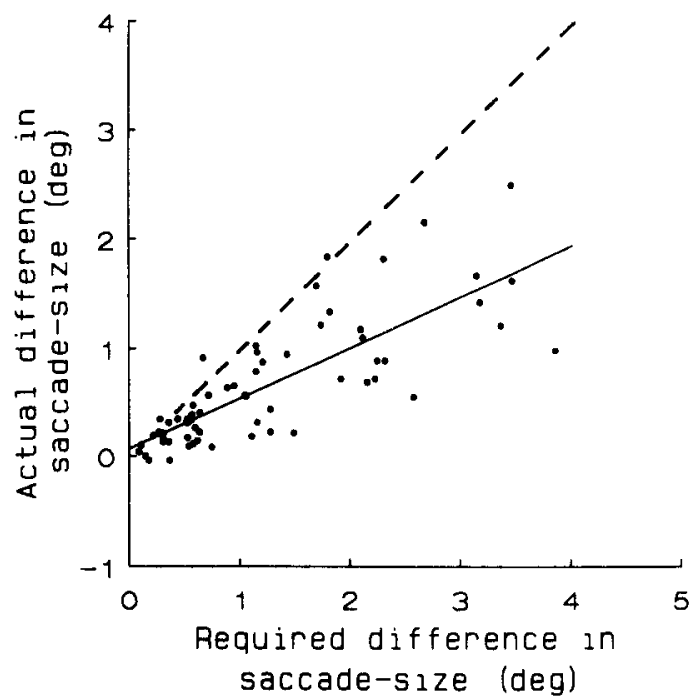

Fig. 3. As Fig. 2, for monocular viewing. The slope of the calculated linear regression line is 0.46 . The results in this figure represent the part of the adaptation that was independent of immediate binocular visual input.

may suggest that the amount of nonconjugate adaptation was, for every individual subject, a fixed percentage of what was called for by the spectacles. However, closer examination of individual data revealed that there was no such fixed relationship within subjects. Figures 2 and 3 also show that the nonconjugate adaptation was more variable with one eye covered than when both eyes were viewing.

Some subjects (HH and PJ) experienced diplopia of the upper targets, notably at larger target eccentricities, which indicates that nonconjugate adaptation was inadequate in the upper oculomotor field. This corresponds well with their large vergence-deficits ( $>1 \mathrm{deg}$ ) associ-

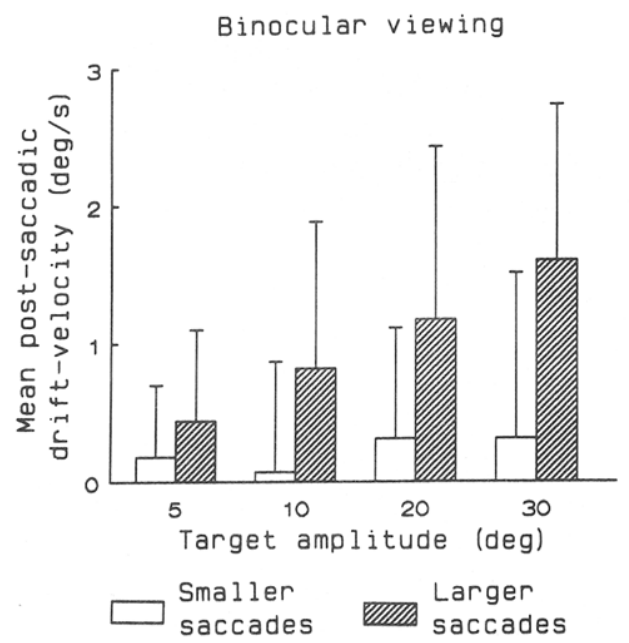

ated with vertical saccades during binocular viewing. Diplopia never occurred for targets in the lower oculomotor fields or targets positioned along the horizontal meridian.

\section{Post-saccadic drift}

In our spectacle-wearers, post-saccadic drift of vertical saccades was not significantly different in the two eyes $\left(F_{1,7}=0.26 ; P>0.5\right)$ and did also not differ significantly from control subjects. Post-saccadic drift of horizontal saccades was, however, asymmetrical. Following horizontal saccades, post-saccadic drift-velocities of the eye that made the larger saccades were higher than the post-saccadic drift-velocities of the eye that made the smaller saccades $\left(F_{1,7}=7.00\right.$; $P<0.05)$. This difference in post-saccadic driftvelocity between the two eyes was larger during binocular viewing than during monocular viewing $\left(F_{2,14}=12.0 ; P<0.001\right)$. It averaged about $0.8 \mathrm{deg} / \mathrm{sec}$ with both eyes viewing and about $0.3 \mathrm{deg} / \mathrm{sec}$ with only one eye viewing (Fig. 4). The direction of the post-saccadic drift of the eye that made the larger horizontal saccade was such that it reduced the vergence-deficit that was present at saccadic offset. As already mentioned, this reduction was most effective during binocular viewing. Similarly to normal, unadapted saccades (Kapoula et al., 1986; Collewijn et al., 1988b), mean post-saccadic drift-velocities increased with the target separation $\left(F_{3,21}=14.2\right.$; $P<0.0005)$. The asymmetry between the postsaccadic drift of the two eyes in our spectaclewearers was, however, in contrast to the situation for normal saccades, independent of the target separation.

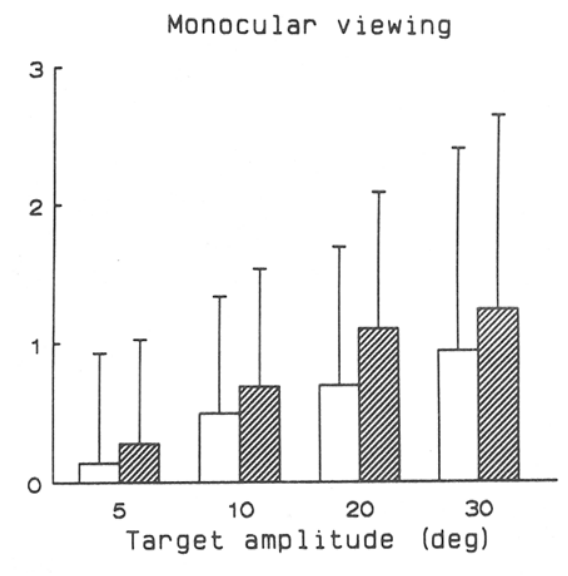

Fig. 4. Mean post-saccadic drift-velocities for all subjects wearing anisometropic spectacles at 4 target amplitudes, plotted for the eye that made the smaller saccades and for the fellow eye. Left panel: binocular viewing; right panel: monocular viewing. Bars represent ISD. 
In our control subjects we observed the nasotemporal asymmetries of post-saccadic drift that have been described before for normal saccades (Kapoula et al., 1986; Collewijn et al., 1988b). Horizontal, adducting saccades were followed by an onward drift with a velocity of about $0.8 \mathrm{deg} / \mathrm{sec}$ increasing in velocity with larger target separations. This velocity was higher by about $0.3 \mathrm{deg} / \mathrm{sec}$ when the fellow eye was covered. Abducting saccades were associated with a post-saccadic drift of lower velocity than adducting saccades: it averaged about $0.4 \mathrm{deg} /$ sec. Its direction could be either onward or backward. It became more frequently onward at larger target-separations. Covering one eye affected neither the direction nor the velocity of the post-saccadic drift of normal, abducting saccades. These typical naso-temporal asymmetries in post-saccadic drift of normal, horizontal saccades were not present in our subjects wearing anisometropic spectacles $\left(F_{1,7}=2.59 ; P>0.1\right)$. In other words, post-saccadic drift of nonconjugately adapted saccades was the same for adducting and abducting saccades of each eye.

\section{Smooth pursuit}

When the spectacle-wearers tracked a target that made a uniform, circular movement (dia:
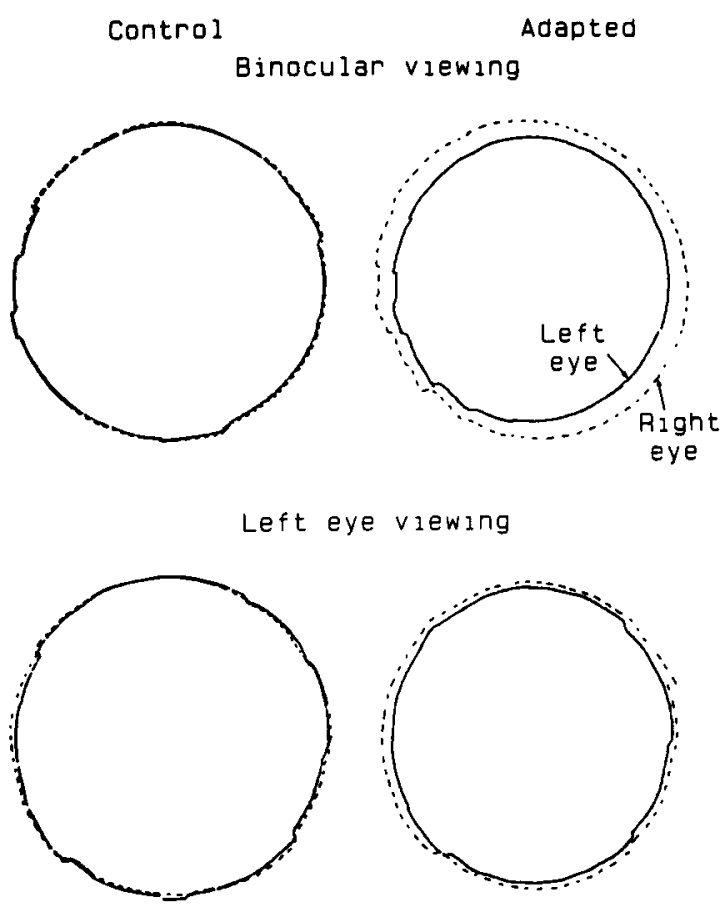

Fig. 5. Binocular recordings of smooth-pursuit eye movements, during binocular viewing (upper panels) and during monocular viewing (left eye viewing, lower panels). Recordings of a control subject are presented in the left panels; recordings of a subject wearing anisometropic spectacles are shown in the right panels.

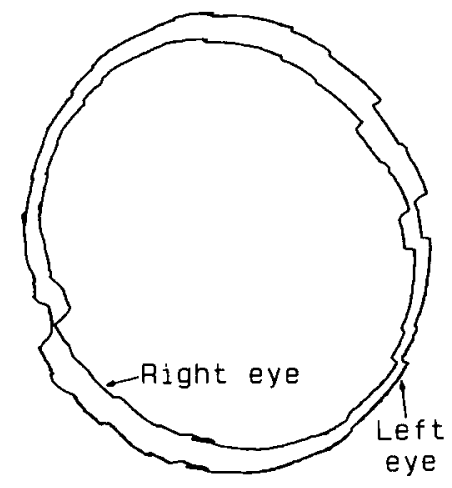

Fig. 6. Binocular recordings made during the smooth-pursult task in a subject with powerful cylindrical lenses (subject $\mathrm{CB}$; right eye cylinder $+5 \mathrm{D}$, axis $65 \mathrm{deg}$ ). Binocular viewing.

$30 \mathrm{deg}$; velocity: about $11 \mathrm{deg} / \mathrm{sec}$ ) the magnitudes of the eye movements were different in the two eyes. This conspicuous nonconjugacy did not occur in our control subjects. Because the nonconjugacies in smooth-pursuit eye movements, observed in the spectacle-wearers, met the requirements of their spectacles, we attribute them to adaptation. Some recordings, typical of either the adapted or the control group, are presented in Fig. 5 (spectacle-wearer: HH). As we were not interested in systematic shifts in gaze due to any prismatic effects of the glasses or two phorias, we have plotted the recordings concentrically. Note that both subjects occasionally made saccades during the execution of the pursuit-task, which is a normal phenomenon. Those subjects who wore spectacles with cylindrical lenses made elliptical eye movements, corresponding with the refractive powers and cylinder-axes of their glasses. One such example (subject CB) is presented in Fig. 6.

To assess the magnitude of the nonconjugate adaptations, we calculated the size-differences between the diameters of the circular trajectories of each eye along the horizontal and vertical meridian in the spectacle-wearers. Adaptive nonconjugacies in diameter of the circular smoothpursuit eye movements could become as large as about $4 \mathrm{deg}$. During binocular viewing, differences in diameter beween movements of the two eyes were larger by about $0.9 \mathrm{deg}$ than during monocular viewing, along either meridian (paired Student's $t$-test: $P<0.05$ ). The nonconjugacies could be meridian-specific: subject PA displayed virtually no asymmetry along the horizontal meridian, whereas the asymmetry was about $2 \mathrm{deg}$ or more along the vertical meridian. Note that this difference between the two meridians was actually imposed by her spectacles. 
To assess the completeness of the adaptations, we computed once again the vergence-deficits, by subtracting the actual differences in diameter-size between the movements of the two eyes from the differences that were required by the spectacles. The smooth-pursuit eye movements turned out to be very well adapted: vergence-deficits during binocular viewing were on the order of $0.1 \mathrm{deg}$. Vergence-deficits along the vertical meridian were, for the group of spectacle-wearers as a whole, not significantly different from those along the horizontal meridian (paired Student's $t$-test: $P>0.05$ ), with either binocular or monocular viewing.

We next compared the vergence-deficits of the nonconjugate smooth-pursuit eye movements to those of the saccades. With both eyes viewing, the vergence-deficits of smooth-pursuit eye movements were smaller by about $0.6 \mathrm{deg}$ than those associated with saccades (horizontal as well as vertical) that were made between targets positioned equally far apart $(30 \mathrm{deg})$ as the diameter of the circular target-movement (paired Student's $t$-test: $P<0.05$ ). As with vertical saccades, subjects $\mathrm{HH}$ and $\mathrm{PJ}$ experienced doubleimages of the target in the upper oculomotor range. During monocular viewing, however, there was no significant difference in vergence- deficit between saccades and smooth-pursuit eye movements.

In conclusion, during binocular viewing, nonconjugate adaptation was more complete for smooth-pursuit eye movements than for saccades. During monocular viewing, however, there was no such difference.

\section{Versatility of nonconjugate adaptation}

Subject PJ repeated the experiments on another day. This time, however, he did not wear his anisometropic spectacles, which he had left off for about $1 \mathrm{hr}$ prior to the experiment. It will be recalled that he normally wears them for only several hours a day, and that his intermittent wearing of his spectacles therefore requires that he either make nonconjugate eye movements or conjugate eye movements, depending on whether he has his glasses on or not. Figure 7 presents recordings of saccades of his two eyes during monocular viewing, both with and without his own spectacles. It is clear from this figure that the size-differences between saccades of the two eyes that were present while he wore his spectacles were markedly reduced when he did not wear them. In both conditions, however, viewing was monocular and therefore contained no direct stimulus for saccades of unequal size.

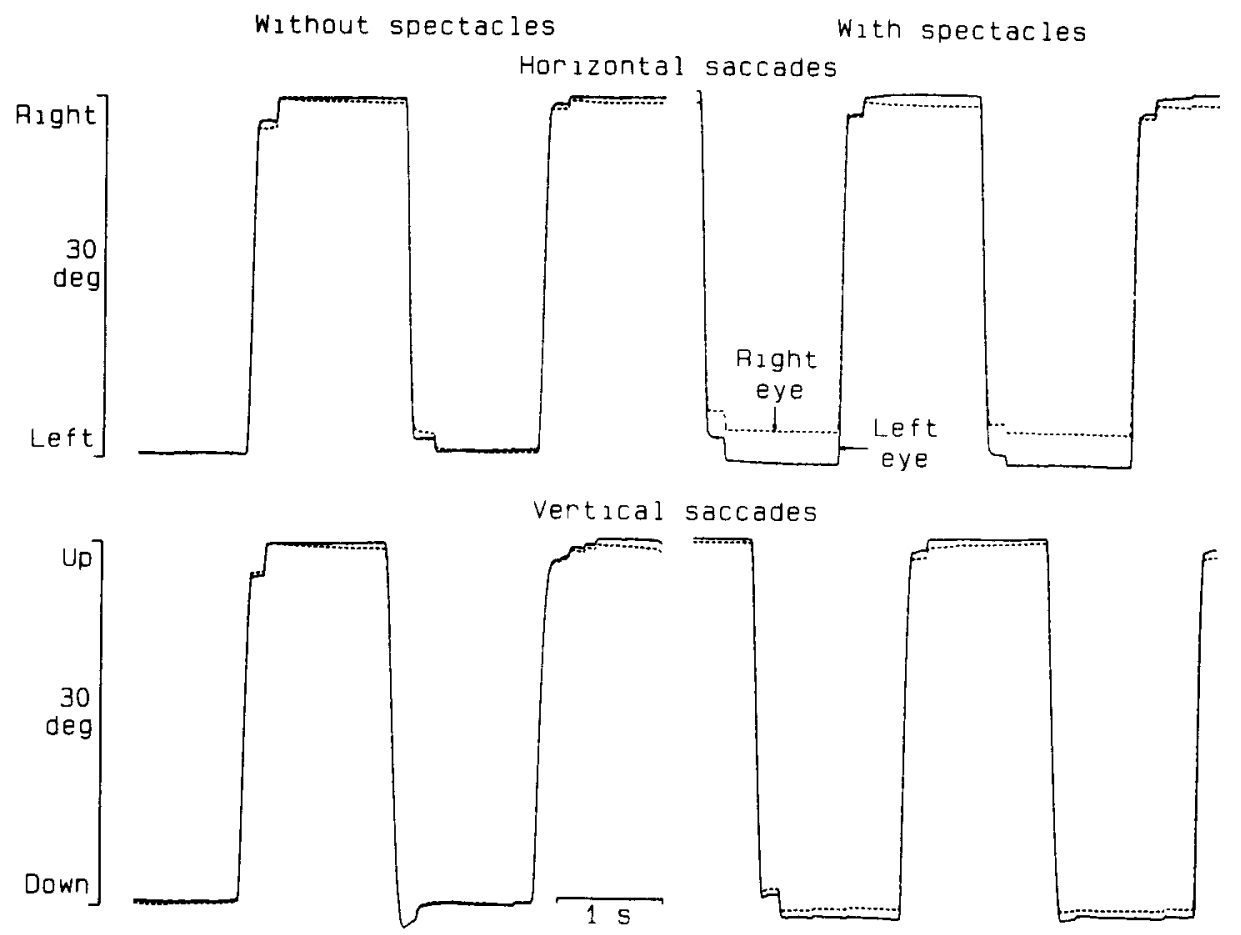

Fig. 7. Recordings of saccades made by subject PJ, who did not wear his spectacles continuously, showing versatility of adaptation. Viewing was monocular with the left eye. Left panels present recordings made after the spectacles had been left off for $1 \mathrm{hr}$. Right panels present recordings made with the subject wearing his own, anisometropic spectacles. 
During binocular viewing, there were no significant size-differences between saccades of the two eyes, except for the usual temporo-nasal asymmetries (Collewijn et al., 1988b). Vertical saccades made with both eyes viewing did not cause double-images of the upper targets, such as occurred with his spectacles on. Mean postsaccadic drift-velocities in the uncorrected condition were somewhat different from when he wore his spectacles. However, these changes showed no consistent pattern. Smooth eye movements, made with one eye covered, in pursuit of the circularly moving target, were smaller in his right eye than in his left eye, which would meet the requirements of his spectacles, although he did not wear them at that time. With both eyes viewing, however, the two eyes made circular movements of equal size.

In conclusion: the intermittent wearing of anisometropic spectacles by this subject was, under normal conditions (binocular viewing), adequately matched by a very versatile degree of nonconjugate adaptation.

\section{DISCUSSION}

The present experiments demonstrate that saccadic eye movements of the two eyes adapt nonconjugately to the long-term wearing of anisometropic spectacles, and thus confirm and extend previous findings by our group (Erkelens et al., 1989). In addition, we found that longterm nonconjugate adaptation was also present in smooth-pursuit eye movements. As a result of nonconjugate adaptation, Hering's law of equal innervation is violated in the sense that these two kinds of (versional) eye movements become unequally large in the two eyes.

Hering (1868) originally observed that the two eyes are so well coordinated that they always look at the same object. Based on this observation, he formulated his law on the so-called equal innervation of the two eyes. Importantly, his law is merely a description of the high degree of yoking of the eyes. It does not relate directly to the actual motor commands (or "innervation") of the two eyes, which may not at all be equal in case of asymmetries in the physical properties of, for instance, the motoneurons, the external eye muscles, or the tissues in which the eyeballs are suspended. Normally, a high degree of conjugacy is desirable as a basic mode of binocular gaze-shifts. In the light of the evidence emerging from investigations such as the present one on the adaptive control of the two eyes separately, it would appear that such conjugacy is obtained and maintained through adaptation, because normal, every-day life conditions exert a certain pressure for such conjugacy. In the absence of such pressure, conjugacy might break down gradually. Indeed, it has been shown in monkeys that conjugacy may deteriorate when one eye is deprived of vision for 1 week (Vilis, Yates \& Hore, 1985; Viirre, Cadera \& Vilis, 1987). Therefore, we postulate that the high degree of normal yoking of the eyes results from the capacity of the oculomotor system to adapt, and does not originate from a fixed and rigid coupling of motor commands which are equal for the two eyes. In case changes occur within the oculomotor system, for example due to pathology or growth, the adaptation will restore conjugacy within a certain adaptive range.

In our current interpretation, conjugacy is operationally defined. Its purpose is the matching between binocular oculomotor metrics to binocular optical metrics. In most normal conditions, this will imply rotation of the eyes through equal mechanical angles. If, however, asymmetrical changes occur in the optical metrics, e.g. by the wearing of anisometropic spectacles, the coordination between the two eyes will be adjusted (also within a certain range) up to the point where yoking becomes functionally adequate. In such a condition, Hering's law still holds in a functional sense, although the eye movements have become nonconjugate.

The functional significance of nonconjugate adaptation is that it prevents the occurrence of diplopia that would otherwise ensure. Erkelens et al. (1989) found, in the subject they reported on, that the nonconjugate adaptation to his long-term wearing of anisometropic spectacles was more complete for vertical saccades than for horizontal saccades. The authors suggested that this meridional difference might be explained by the fact that the fusional limits for vertical disparities are smaller than for horizontal disparities (Fender \& Julesz, 1967; Piantanida, 1986; Erkelens, 1988). Nonconjugate adaptation would then occur up to the level at which fixation disparities were reduced to within the fusional limits. This does not imply, however, that loss of fusion is the appropriate stimulus for nonconjugate adaptation, as will now be argued. In our present experiments, vertical nonconjugate adaptations were, for the group as a whole, equally complete as horizontal nonconjugate adaptations, although some idiosyncratic variations existed. The imperfections of these non- 
conjugate adaptations were, for saccades in either meridian, almost always smaller than $0.75 \mathrm{deg}$ during binocular viewing, which is, for horizontal saccades, well within the fusional limit, which can be as large as about $2 \mathrm{deg}$ (Erkelens, 1988). Therefore, for horizontal saccades, loss of fusion does not appear to be the appropriate stimulus for nonconjugate adaptation. Along the vertical meridian, which has narrower fusional limits, this appears to hold also, because we found no better nonconjugate adaptation of vertical saccades than of horizontal saccades. Furthermore, if diplopia at saccadic offset were the adequate stimulus for nonconjugate adaptation, we would presumably experience double-images very frequently throughout the course of a lifetime, before nonconjugate adaptations to local changes within the oculomotor system, caused by ageing, disease or fatigue, would come about. Consistent fixationdisparities, within the fusional range, present at saccadic offset, are a more probable candidate for such a stimulus. We would argue that fixation-disparity is a more direct stimulus, because of its relative simplicity, compared to fusion, or the loss of it, which involves higher visual processing.

We found no uniform percentage of nonconjugate adaptation at the various target separations. This suggests that nonconjugate adaptation does not result from the resetting of a few general gain parameters that control the coordination of the two eyes. Such a general resetting has been proposed as a possible control strategy for adaptation of the saccadic subsystem (e.g. Deubel, 1986). Although some of the variability in our present experiments may be due to noise, our results suggest that nonconjugate adaptation occurs on a point-to-point basis on the topology of sensory-motor maps, in such a way that the vergence-deficits, or fixation disparities during binocular viewing, remain within narrow limits. A specific argument against parametric adaptation seems to be that two of our subjects experienced double-images in the upper oculomotor range, secondary to poor nonconjugate adaptation in that range. In case of parametric adjustment, the adaptation would have been equally large anywhere in the oculomotor range. A point-to-point adaptation, based on experience, might better explain the poorer adaptation in the upper oculomotor range because eye movements are normally made more frequently in the lower than in the upper oculomotor range. A similar explanation was also given by
Henson and Dharamshi (1982), who found that adaptation of vertical phorias to anisometropic spectacles was larger, and also more rapid, in the lower oculomotor range than in the upper oculomotor range. This phenomenon had also been described by Ellerbrock (1948). Our two subjects did not experience double-images when they took off their glasses; this excludes restrictive pathology in the upper oculomotor range.

Why is nonconjugate adaptation more complete during binocular viewing than during monocular viewing? An obvious explanation is that, during binocular viewing, both eyes receive direct visual information on the position of the newly selected target and, therefore, accurate motor commands can be computed for each individual eye. With one eye covered, this is obviously not the case. Our results therefore suggest that the control of nonconjugate coordination of saccades of the two eyes consists of, at least, two components: (1) a fairly steady, hard-programmed interocular coordination, demonstrable during monocular viewing, which is not complete, but forms a basic level of interocular coordination, and which can only be modified slowly and through experience, and (2) a component that is complementary in action to the first one, and that requires direct, binocular visual information for the fine-tuning of accurate coordination of the two eyes. The hardprogrammed component would presumably be too robust to meet the requirements of interocular coordination in normal life, since most objects we look at vary both in both direction and in distance, thus requiring slight departures from the basic interocular coordination of saccades. The second, fine-tuning component would probably have a relatively small range. Support for such a limited range of the second component comes from the common experience that when a subject puts on anisometropic spectacles for the first time, he will perceive double-images. Only through continued experience will he be able to maintain fusion at all times. Adaptation will only be adequate if the contribution of one component is sufficiently complemented by the other.

Another striking feature of the nonconjugate adaptation of saccades to the wearing of anisometropic spectacles is that the degree of adaptation can be very versatile. This was demonstrated by the difference in nonconjugate adaptation in subject $P J$ either with and without his spectacles on. This difference was not only present during binocular viewing, but also during monocular 
viewing, which shows that he may change his hard-programmed nonconjugate adaptation very quickly. We do not know whether his hardprogrammed nonconjugate adaptation can vary so rapidly between only two, more or less remembered, modalities, or whether it can vary equally rapidly over a wider range to meet any requirement.

The nonconjugate changes in post-saccadic drift were very small and restricted to horizontal saccades. Although small, they were nonetheless very distinct, and tended to eliminate residual disparities, left by the incomplete nonconjugate adaptation of the main part of the saccades. These drifts presumably reflect asymmetrical, adaptive resettings of the pulse-step ratios that characterize the motor commands to the external eye-muscles for the generation of saccades. These very slight, asymmetrical changes in postsaccadic drift-velocities contrast with the dramatic changes in post-saccadic drift associated with palsies of one or more external eye-muscles (Kommerell et al., 1976; Abel et al., 1978; Snow et al., 1985; Optican \& Robinson, 1980; Optican et al., 1985). Despite the similarity of both conditions in requiring an asymmetrical resetting of the pulse and step motor commands, there are also dissimilarities in the pressure for such adaptation, which might possibly explain the differences in post-saccadic drift. One obvious difference is, that the eye-muscles of our subjects were not affected. Therefore, the equilibrium between agonists and antagonists was not disrupted, as with one paretic muscle. Because of these, and perhaps other, dissimilarities in the stimulus for nonconjugate adaptation, it is possible that both conditions address essentially different processes of adaptation within the saccadic subsystem, which is reflected by differences in post-saccadic drift. As post-saccadic drift may adapt independently of saccades (Optican \& Miles, 1985), it might also be that the observed differences in post-saccadic drift result only from different adaptations of the post-saccadic drift per se, and not from different plastic changes of the entire programming of saccades.

Only limited data exist on adaptive processes of smooth-pursuit eye movements. Nonconjugate, short-term adaptations of smooth-pursuit eye movements have been described before by Horner et al. (1988) and, more extensively, by Schor et al. (1990). An important feature of smooth-pursuit eye movements is that the movements are relatively slow, which allows continuous visual feedback. In contrast, saccades are too fast to be controlled by continuous visual feedback. The continuous visual feedback that takes place during smooth-pursuit eye movements may possibly explain why the vergencedeficits that we observed during binocular viewing were smaller when our subjects tracked the slowly moving target than when they made saccades. However, this difference in vergencedeficits can also be partly explained by the fact that saccades usually undershoot their target and are then followed by one or more secondary saccades which in turn may reduce the vergencedeficits, present in the offset of the primary saccades. In addition, post-saccadic drift of horizontal saccades also reduced the vergence-deficits.

More significantly, nonconjugate adaptations of smooth-pursuit eye movements were also present when one eye was covered. The vergencedeficits, used as a measure of nonconjugate adaptation, were in this case larger than those during binocular viewing, but equal to those associated with saccades made during monocular viewing. One may therefore speculate that nonconjugate adaptation of smooth-pursuit eye movements occurs in a way similar to that for the saccadic subsystem, i.e. by a hardprogrammed fairly robust and incomplete component, as well as by a complementary, fine-tuning component, that requires direct, binocular visual information. In the present experiments, the hard-wired component was quantitatively identical for smooth-pursuit and for saccades. The nature of our experiments, however, does not allow us to speculate on the possibility of structures, shared by both eye movement subsystems, that control interocular coordination, because the adaptive pressure in our subjects was not different for saccades and pursuit. Recent experiments by Schor et al. (1990) have shown the possibility of selective nonconjugate adaptation of either saccades or pursuit, by the use of suitably different training conditions.

In conclusion, long-term nonconjugate adaptation to anisometropic spectacles occurs adequately within the saccadic subsystem, both with respect to the pulse and the step of the oculomotor commands, and also in the smoothpursuit subsystem. A drawback of the present experiment was that the lens-prescriptions were very heterogeneous. Experiments which focus on short-term nonconjugate adaptations to standard anisometropic spectacles will be reported in the accompanying paper (Lemij \& Collewijn, 1991). 
Acknowledgements-This research was supported by the foundation for Medical Research MEDIGON (The Netherlands), Grant No. 900-550-092. We thank Drs C. J Erkelens and J. van der Steen for writing the necessary computer programmes, and for their invaluable comments.

\section{REFERENCES}

Abel, L. A., Schmıdt, D., Dell'Osso. L. F. \& Daroff, R. B (1978). Saccadic system plasticity in humans Annals of Neurology, 4, 313-318.

Albano, J. E. \& King, W. M. (1989). Rapıd adaptation of saccadic amplitude in humans and monkeys. Investigative Ophthalmology and Visual Science, 30, 1883-1893

Alpern, M. (1962). Specification of the direction of regard In Davson, H. (Ed.). The eye, Vol 3 (p. 12). New York Academic Press.

Bahill, T. A., Clark, M. R. \& Stark, L. (1975). Dynamıc overshoot in saccadic eye movements is caused by neurological control signal reversals. Experimental Neurology. $48,107-122$.

Bennett. A. G. \& Francis, J. L. (1962). Ametropia and its correction. In Davson, H. (Ed.). The eve. Vol 4 (pp. 174-176). New York: Academic Press.

Carpenter, R. H. S. (1988). Movements of the eyes London: Pion.

Collewijn, H., Erkelens, C. J. \& Stemman, R M (1988a). Undoing Hering's law: Differential adaptation of saccadic amplitude in the two eyes. Investigatwe Ophthalmology and Visual Science, 29, 166.

Collewijn, H., Erkelens, C J \& Steınman, R. M. (1988b) Binocular co-ordination of human horizontal saccadic eye movements. Journal of Phystology, 404, 157-182.

Collewijn, H., Erkelens, C. J. \& Steınman, R. M. (1988c) Binocular co-ordination of human vertical saccadic eye movements. Journal of Physiology, 404. 183-197.

Collewijn, H., van der Mark, F \& Jansen, T C. (1975), Precise recording of human eye movements Vision Research, 15, 447-450

Collewijn, H., van der Steen, J. \& Steinman, R. M. (1985) Human eye movements associated with blinks and prolonged eyelid closure. Journal of Neurophysiology. 54, 11-27.

Deubel, H. (1987). Adaptivity of gain and direction in oblique saccades. In O'Regan, J. K. \& Lévy-Schoen, A. (Eds), Eye movements. From phystology to cognitton (pp. 181-190). Amsterdam. Elsevier

Deubel, H., Wolf, W. \& Hauske, G. (1986). Adaptıve gain control of saccadic eye movements Human Neurobiology, $5,245-253$.

Ellerbrock, V. J. (1948). Further study of effects induced by anisometropic corrections. American Journal of Optometry and Archices of American Academy of Optometry, 25. 430-437.

Erkelens, C. J. (1988). Fusional limits for a large randomdot stereogram. Vision Research, 28, 345-353

Erkelens, C. J., Collewijn, H. \& Steinman, R. M. (1989) Asymmetrical adaptation of human saccades to anisometropic spectacles. Investigatize Ophthalmology and Visual Science, 30, 1132-1145.

Fender, D. \& Julesz. B. (1967). Extension of Panum's fusional area in binocularly stabilized vision. Journal of the Optical Society of America, 57, 819-830

Henson, D. B. \& Dharamshı, B. G (1982) Oculomotor adaptation to induced heterophoria and anisometropia. Investigattue Ophthalmology and Visual Sctence, 22, 234-240.
Hering, E. (1868). Die Lehre vom binokularen Sehen. English translation in Bridgeman, B. \& Stark, L. (Eds) (1977), The theory of binocular vision. New York: Plenum Press.

Horner, D. G., Gleason, G. \& Schor, C. M. (1988). The recalibration of Hering's law for versional eye movements in response to aniseikonia. Investigative Ophthalmology and Visual Science, 29, 136.

Kapoula, Z. A., Robinson, D. A. \& Hain, T. C. (1986). Motion of the eye immediately after a saccade. Experimental Brain Research, 61, 386-394.

Kommerell, G., Olivier, D. \& Theopold, H. (1976). Adaptive programming of phasic and tonic components in saccadic eye movements. Investigations in patients with abducens palsy Investigative Ophthalmology, 15, 657-660.

Lemij, H G. (1990). Asymmetrical adaptation of human saccades to anisometropic spectacles. Doctoral thesis, Erasmus University, Rotterdam, The Netherlands.

Lemij, H. G. \& Collewijn, H. (1989). Differences in accuracy of human saccades between stationary and jumping targets. Vision Research, 29, 1737-1748.

Lemij, H. G. \& Collewijn, H. (1991). Short-term nonconjugate adaptation of human saccades to anisometropic spectacles. Vision Research, 31, 1955-1966.

Levi, L., Zee, D. S., Hain, T. C., Fletcher, W. A. \& Miller, N. R. (1988). Ocular motor adaptation to anisometropic correction. Investigative Ophthalmology and Visual Science, 29, 167

Miller, J. M., Anstis, T. \& Templeton, W. B. (1981) Saccadic plasticity: Parametric adaptive control by retinal feedback. Journal of Experimental Psychology: Human Perception and Performance, 7, 356-366.

Oohira, A., Zee, D. S. \& Guyton, D. L. (1991). Disconjugate adaptation to long-standing, large-amplitude spectaclecorrected anisometropia. Investigative Ophthalmology and Visual Sclence, 32, 1693-1703.

Optican, L. M. \& Miles, F. A. (1985). Visually induced adaptıve changes in primate saccadic oculomotor control signals. Journal of Neurophysiology, 54, 940-958.

Optican, L M. \& Robinson, D. A. (1980). Cerebellardependent adaptive control of primate saccadic system. Journal of Neurophysiology, 44, 1058-1076.

Optican, L. M., Zee, D. S. \& Chu, F. C. (1985). Adaptive response to ocular muscle weakness in human pursuit and saccadic eye movements. Journal of Neurophysiology, 54, $110-122$

Piantanida. T. P. (1986). Stereo hysteresis revisited. Vision Research, 26, 431-437.

Robinson, D. A. (1963). A method of measuring eye movements using a scleral search coil in a magnetic field. The Institute of Electrical and Electronics Engineers, Inc., Transactions on Biomedical Engineering, BME-10, 137-145.

Schor. C M.. Gleason, G. \& Horner, D. (1988). The variability and adaptability of Hering's law for yoked reflexive eye movements. Investigative Ophthalmology and Visual Science, 29, 136.

Schor, C. M., Gleason, G. \& Horner, D. (1990). Selective nonconjugate binocular adaptation of vertical saccades and pursuits. Vision Research, 30, 1827-1844.

Snow, R., Hore, J. \& Vilis, T. (1985). Adaptation of saccadic and vestibulo-ocular systems after extraocular muscle tenectomy. Investigative Ophthalmology and Visual Science, 26, $924-931$

Viirre, E., Cadera, W. \& Vilis, T. (1987). The pattern of changes produced in the saccadic system and vestibuloocular reflex by visually patching one eye. Journal of Neurophysiology, 57, 92-103. 
Viirre, E., Cadera, W. \& Vilis, T. (1988). Monocular adaptation of the saccadic system and vestibulo-ocular reflex. Investigative Ophthalmology and Visual Science, 29. 1339-1347.

Vilis, T., Yates, S. \& Hore, J. (1985). Visual patching of one eye produces changes in saccadic properties in the unseeing eye. Developmental Brain Research, 17, 290-292.

Zee, D. S. \& Levi, L. (1989). Neurological aspects of vergence eye movements. Revue Neurologique (Paris), 145 , 613-620. 